\title{
Analysis of the Lotus japonicus nuclear pore NUP107-160 subcomplex reveals pronounced structural plasticity and functional redundancy
}

\author{
Andreas Binder and Martin Parniske* \\ Faculty of Biology, Genetics, University of Munich, Martinsried, Germany
}

Edited by:

Katja Graumann, Oxford Brookes

University, UK

\section{Reviewed by:}

Kentaro Tamura, Kyoto University, Japan

Geraint Parry, University of

Liverpool, UK

\section{*Correspondence:}

Martin Parniske, Faculty of Biology Genetics, University of Munich, Großhaderner Straße 4, 82152 Martinsried, Germany

e-mail:parniske@Imu.de
Mutations in the Lotus japonicus nucleoporin genes, NUP85, NUP133, and NENA (SEH1), lead to defects in plant-microbe symbiotic signaling. The homologous proteins in yeast and vertebrates are part of the conserved NUP84/NUP107-160 subcomplex, which is an essential component of the nuclear pore scaffold and has a pivotal role in nuclear pore complex (NPC) assembly. Loss and down-regulation of NUP84/NUP107-160 members has previously been correlated with a variety of growth and molecular defects, however, in L. japonicus only surprisingly specific phenotypes have been reported. We investigated whether Lotus nup85, nup 133, and nena mutants exhibit general defects in NPC composition and distribution. Whole mount immunolocalization confirmed a typical nucleoporin-like localization for NUP133, which was unchanged in the nup85-1 mutant. Severe NPC clustering and aberrations in the nuclear envelope have been reported for Saccharomyces cerevisiae nup85 and nup133 mutants. However, upon transmission electron microscopy analysis of L. japonicus nup85, nup 133 and nena, we detected only a slight reduction in the average distances between neighboring NPCs in nup133. Using quantitative immunodetection on protein-blots we observed that loss of individual nucleoporins affected the protein levels of other NUP107-160 complex members. Unlike the single mutants, nup85/nup133 double mutants exhibited severe temperature dependent growth and developmental defects, suggesting that the loss of more than one NUP107-160 member affects basal functions of the NPC.

Keywords: nuclear pore complex, nucleoporins, Lotus japonicus, NUP107-160 subcomplex, plant nucleus

\section{INTRODUCTION}

Eukaryotic cells are organized into membrane enclosed organelles, allowing for functional and spatial division of cellular processes. The nucleus is confined by a double membrane, the nuclear envelope, thus separating nuclear DNA from cytoplasm and uncoupling transcription and translation. Exchange of macromolecules in and out of the nucleus is mediated by nuclear pore complexes (NPCs), which are embedded into the nuclear membranes (Aitchison and Rout, 2012; Grossman et al., 2012). NPCs are large supramolecular assemblies, composed of about 30 proteins, termed nucleoporins (NUPs), which form stable subcomplexes. Each NUP is present in multiple copies due to the NPCs eight fold rotational symmetry and an imperfect symmetry between cytosolic and nucleoplasmic side (Alber et al., 2007; Hoelz et al., 2011). In addition to their role as gatekeepers in nucleocytoplasmic transport, NPCs and individual NUPs are involved in a large number of physiological processes, including kinetochore and spindle assembly, cell cycle control, regulation of gene expression, chromatin organization, DNA repair, and DNA replication (Capelson and Hetzer, 2009; Capelson et al., 2010; Strambio-De-Castillia et al., 2010; Wozniak et al., 2010; Van De Vosse et al., 2011, 2013; Bukata et al., 2013).
Nuclear pore components were mostly studied in yeast and vertebrates (Hoelz et al., 2011; Aitchison and Rout, 2012; Grossman et al., 2012), however relatively little is known about them in plants. A recent proteomics study in A. thaliana identified and characterized at least 29 nucleoporins, 22 of which were previously not annotated in plants (Tamura et al., 2010). Considering sequence similarity and domain organization, the plant proteins were more closely related to the vertebrate than to the yeast orthologs. For six vertebrate NUPs (NUP358, NUP188, NUP153, NUP45, NUP37, POM121), no homologs were found in A. thaliana, while one A. thaliana nucleoporin, NUP136/NUP1 was unique to plants, suggesting specific adaptations of the plant nuclear pore to particular aspects of plant cell biology (Tamura et al., 2010). The overall structure of the plant NPC resembles that of other eukaryotes (Fiserova et al., 2009), with an approximate diameter of $105 \mathrm{~nm}$ in Nicotiana tobacum, it is more similar to the vertebrate NPC (110-120 nm in Xenopus; Goldberg and Allen, 1996) than the smaller Saccharomyces cerevisiae NPC (95 nm; Kiseleva et al., 2004).

Forward and reverse genetic analyses implicated plant nucleoporins in plant microbe interactions, development, flowering, hormone- and cold stress signaling (Binder and Parniske, 2013). The majority of the genetically identified plant NUPs 
are homologous to members of the conserved nuclear pore NUP107-160 (in vertebrates) or NUP84 (in yeast) subcomplex. The complex forms a Y-shaped structure that is composed of at least seven core nucleoporins (NUP85, NUP96, NUP107, NUP133, NUP160, SEH1, SEC13; Belgareh et al., 2001; Lutzmann et al., 2002; Harel et al., 2003; Walther et al., 2003) and can contain up to three additional proteins (NUP37, NUP43, and ELYS) depending on the organism (Loïodice et al., 2004; Rasala et al., 2006). While the subcomplex has not been fully characterized in plants, it is likely conserved, as homologous genes of all core yeast NUP84 and vertebrate NUP107-160 members are present in Arabidopsis (Wiermer et al., 2012). Additionally, positive interaction of L. japonicus NUP85 and NENA (SEH1) in yeast-two-hybrid analysis is consistent with the arrangement of the homologous proteins in the S. cerevisiae NUP84 complex (Lutzmann et al., 2002; Groth et al., 2010). The subcomplex is the largest of the nuclear pore subunits and an essential structural component, required for nuclear pore assembly (Harel et al., 2003; Walther et al., 2003; Doucet et al., 2010). During mitosis it is recruited to kinetochores and contributes to normal kinetochore functions including spindle assembly (Belgareh et al., 2001; Orjalo et al., 2006; Zuccolo et al., 2007). Loss or depletion of individual NUP107-160/NUP84 NUPs is associated with different phenotypes. In S. cerevisiae, mutations in NUP84 members lead to temperature dependent growth defects, aberrant NPC biogenesis and distribution as well as accumulation of nuclear polyA RNA (Doye et al., 1994; Aitchison et al., 1995; Heath et al., 1995; Li et al., 1995; Pemberton et al., 1995; Siniossoglou et al., 1996; Fernandez-Martinez et al., 2012). In mice, NUP133 is dispensable for many essential NPC functions, however nup133 mutants are disturbed in embryonic neural differentiation (Lupu et al., 2008). In contrast, loss of mouse NUP96 is lethal and low expression levels of NUP96 in heterozygous NUP96 ${ }^{+/}-$individuals affect interferon mediated immune responses (Faria et al., 2006). Mutations in Arabidopsis thaliana NUP96, NUP160 and to a lesser extend in SEH1 also lead to defects in basal and resistance (R)-gene mediated defense (Zhang and Li, 2005; Cheng et al., 2009; Wiermer et al., 2012). Additionally NUP96 and NUP160 are involved in auxin signaling (Parry et al., 2006) and nup160 mutants are hypersensitive to ethylene (via the auxin pathway; Robles et al., 2012) and cold stress (Dong et al., 2006). Both nup96 and nup160 plants flower early and exhibit developmental defects (Parry et al., 2006). In Lotus japonicus three putative NUP107-160 complex nucleoporins, NUP85, NUP133, and the SEH1 homolog NENA are involved in the establishment of plant-microbe symbiosis. Mutations in the NUP genes disturb nuclear calcium spiking, an early physiological response in symbiotic signaling, and cause temperature dependent defects in arbuscular mycorrhiza (AM) and root nodule symbiosis (RNS) (Kistner et al., 2005; Kanamori et al., 2006; Saito et al., 2007; Groth et al., 2010). Furthermore, L. japonicus nup85 mutants show defects in pollen tube growth and both nup85 and nup133 display a slight reduction in seed yield, which could not be observed for nena. However, no obvious additional growth or developmental phenotypes were reported for either of the mutants (Kanamori et al., 2006; Saito et al., 2007; Groth et al., 2010).
The lack of broad pleotropic defects at the plant level in the Lotus nup mutants prompted us to investigate potential changes in the nuclear organization with regard to NPC distribution and nuclear envelope structure. Based on the shared phenotypes of nup85, nup133, and nena mutants in plant-microbe symbiosis, we hypothesized that alterations in the NUP107-160 subcomplex could be responsible for the defects in symbiotic signaling. Investigation of the Lotus nup mutants for altered levels of NUP85 and NUP133 indicated that the loss of individual nucleoporins could lead to changes in the NUP107-160 subcomplex composition and thereby potentially result in the observed phenotypes.

The study of nup85/nup133 double mutants revealed severe defects in growth and development, suggesting that the plant NPC cannot compensate effectively for the loss of more than one of these structural NUPs.

\section{MATERIALS AND METHODS PLANT MATERIAL AND GROWTH}

Lotus japonicus ecotype B-129 Gifu was used as wild type. Seeds were scarified with sand paper and surface sterilized with $2 \%$ $\mathrm{NaClO}+0.1 \%$ SDS. Seedlings were cultivated on $0.8 \%$ Bacto Agar (BD) at 18 or $24^{\circ} \mathrm{C}$ in $16 \mathrm{~h} \mathrm{light} / 8 \mathrm{~h}$ dark cycles.

\section{CROSSING}

L. japonicus flowers of the right stage (containing ripe anthers with unreleased pollen and a straight style) were selected and carefully emasculated by removing petals and anthers with forceps, without releasing the pollen (Jiang and Gresshoff, 1997). Pollen was harvested from the donor plant and released onto the thumb nail. The stigma of the emasculated flower was gently pressed into the pollen. The flower was covered with a plastic tube, which was stuffed with slightly moist cotton at the bottom for protection and to prevent cross-pollination.

\section{GENOTYPING}

L. japonicus nup85 and nup133 crosses and their progeny were genotyped either by (derived) cleaved amplified polymorphism analysis (CAPs/dCAPs) or via the KASP system (LGC genomics). CAPs/dCAPs PCR was performed using 10-50 ng of genomic L. japonicus DNA as a template. For genotyping of NUP85 primers Nup85-1_dCAP_FW (TAAGGTGTTGTTTGTTTCTATTTTATAGCCATG) and Nup85-1_dCAP_Rv(GAAAATGAGAAACATACAACTCAATCC), for genotyping of NUP133 primers Nup133-1_CAPs_FW (TTCTGGCATTCATCGAACAG) and Nup133-1_CAPs_RV (TTTGACCAGCCAGATCCTTC) were used. PCR products were digested with restriction enzymes (NEB) NcoI-HF for NUP85 and RsaI for NUP133 and the products were separated on a 3\% agarose gel. In case of NUP85, the wild type allele resulted in two bands of 30 and $177 \mathrm{bp}$ and the nup85-5 allele in a single band of $207 \mathrm{bp}$. In case of NUP133, the wild type allele showed two bands at 92 and $198 \mathrm{bp}$ and the nup133-1 allele a single band at 290 bp. KASP genotyping PCR was performed according to the manufacturer's instructions with specific primer sets for discrimination of wild type and nup85/nup133 mutant alleles. KASP primers were designed by LGC genomics. Successful crosses and identified double mutants were confirmed by sequencing. 


\section{QUANTITATIVE IMMUNOBLOT}

For production of peptide antibodies, peptides were selected that had no or low homology to other known proteins and contained predicted exposed epitopes, preferentially with several positively or negatively charged amino acids $(\mathrm{R}, \mathrm{H}, \mathrm{K}, \mathrm{D}, \mathrm{E})$ to increase the potential binding avidity of the antibodies. Among the suitable sequences one peptide per protein was selected that contained few or no predicted phosphorylation and N-glycosylation sites. $\alpha$-NUP85 peptide antibodies were raised in guinea-pigs against the peptide $\mathrm{H}_{2} \mathrm{~N}$-LHKYRDFKKSLQQVSGGK- $-\mathrm{CONH}_{2}$ and $\alpha$-NUP133 peptide antibodies were raised in rabbits against the peptide $\mathrm{H}_{2} \mathrm{~N}$-MHLPPEGGDSGQLEGNKGYPR- $\mathrm{CONH}_{2}$. Antibodies were produced by Pineda Antibody Service (Berlin). For total protein extraction two week old L. japonicus roots were ground in liquid nitrogen and taken up $(100 \mathrm{mg} / 200 \mu \mathrm{l})$ in denaturating extraction buffer (10 mM EDTA, $50 \mathrm{mM}$ Hepes, $150 \mathrm{mM}$ $\mathrm{NaCl}, 10 \%$ Sucrose, $5 \mathrm{M}$ Urea, $2 \mathrm{M}$ Thiourea, 1.5\% Triton-X 100, 1\% SDS, 2 mM DTT, 1 mM PMSF, Sigma protease inhibitor cocktail P9599; Sigma-Aldrich). The solution was incubated for $1 \mathrm{~h}$ at $37^{\circ} \mathrm{C}$. Proteins were separated on a polyacrylamide SDS gel and transferred for $3 \mathrm{~h}$ at $30 \mathrm{~V}$ and $1 \mathrm{~h}$ at $100 \mathrm{~V}$ onto a low-background Immobilon FL PVDF membrane (Millipore). Membranes were blocked in 5\% skim milk in TBS $(+0.1 \%$ Tween 20$)$ and incubated overnight at $4^{\circ} \mathrm{C}$ in the primary antibodies and for $2 \mathrm{~h}$ in the secondary antibodies, both diluted in TBS (+3\% skim milk and $0.1 \%$ Tween 20). Primary $\alpha$-NUP85 (Guinea-Pig) antibody was used at a dilution of 1:2000, $\alpha$-NUP133 (rabbit) at 1:5000 and $\alpha$-Histone H3 (mouse; ab1791; abcam) at 1:500. Secondary $\alpha$-guinea-PIG 680 LT (LI-COR) and secondary $\alpha$-rabbit 680 LT (LI-COR) antibodies were diluted to 1:20000, secondary $\alpha$-mouse 800 (Biomol) to 1:10000. Quantification and image acquisition of the secondary antibodies were performed with a LI-COR Odyssey Infrared imager using the Odyssey software.

\section{WHOLE-MOUNT IMMUNOLOCALIZATION}

Immunolocalization of L. japonicus root tissue was performed based on Sauer et al. (2006). L. japonicus plants were grown for 2 weeks on $0.8 \%$ water agar. Root tips $(0.3-0.5 \mathrm{~cm})$ were cut off with a razor blade, placed in freshly prepared fixative solution (4\% Paraformaldehyde dissolved in $1 \times$ PBS with $0.1 \%$ Triton X-100) and vacuum infiltrated for $1 \mathrm{~h}$. Following removal of the fixative, the plant tissue was washed four times for $10 \mathrm{~min}$ with $1 \times$ PBS. Root tips were placed on a small droplet of $\mathrm{ddH}_{2} \mathrm{O}$ on SuperFrost ${ }^{\circledR}$ Plus adhesive slides (Menzel-Gläser) and dried overnight at room temperature (RT). The plant tissue was rehydrated with a drop of $1 \times$ PBS and placed in a humid chamber. 2\% Driselase (D8037-1G; Sigma-Aldrich) was added for cell wall digestion and roots were incubated for $60 \mathrm{~min}$ at $37^{\circ} \mathrm{C}$. After washing with $1 \times$ PBS for six times, 3\% IGEPAL CA-630 (Sigma-Aldrich) with 10\% DMSO was added. These and successive washing steps were carried out in an excess volume of 1 $\times$ PBS in microscopy slide holders. For permeabilization, roots were incubated for $60 \mathrm{~min}$ at RT, then washed five more times with $1 \times$ PBS. As blocking solution 3\% albumin fraction V (BSA; Sigma-Aldrich) in $1 \times$ PBS $(100-200 \mu \mathrm{l})$ was added for $60 \mathrm{~min}$ at RT. After removal of the BSA solution, primary antibodies $(\alpha-$ NUP133; 1:500) were added in 3\% BSA solution, in a sufficient amount to fully cover the roots. Slides were placed in a humid chamber and incubated for $240 \mathrm{~min}$ at $37^{\circ} \mathrm{C}$, then overnight at $4^{\circ} \mathrm{C}$. The samples were washed six times in $1 \times$ PBS. Secondary antibody (Alexa Fluor ${ }^{\circledR} 488 \alpha$-rabbit; Life Technologies) in 3\% BSA was added and roots were incubated for another $180 \mathrm{~min}$ at $37^{\circ} \mathrm{C}$. Slides were again washed for five times in $1 \times$ PBS. To visualize the nuclei $1 \mu \mathrm{g} / \mathrm{ml} 4^{\prime}$,6-Diamidino-2-phenylindole dihydrochloride (DAPI; Sigma-Aldrich) solution diluted in $\mathrm{ddH}_{2} \mathrm{O}$ was added for $10 \mathrm{~min}$. Finally the root tips were washed three times with $\mathrm{ddH}_{2} \mathrm{O}$ to remove excess DAPI and salt. The samples were mounted in 0.25\% 1,4-Diazabicyclo[2.2.2] octan (DABCO; Sigma-Aldrich), $90 \%$ glycerol in $1 \times \mathrm{PBS}$ and stored at $4^{\circ} \mathrm{C}$ in the dark.

Samples were analyzed by 3D structured illumination microscopy on a DeltaVision OMX (Applied precision) microscopic imaging system with a plan-apochromatic $100 \times, 1.4 \mathrm{NA}$, oil-immersion objective (Olympus). A 405 laser and a 488 laser were used to excite the DAPI stained DNA and Alexa ${ }^{\circledR}$ Fluor 488 labeled secondary antibodies, respectively. ImageJ software was used for image processing and generation of Z-stacks.

\section{ELECTRON MICROSCOPY AND NPC DISTANCE QUANTIFICATION}

Root tips of 10 days-old L. japonicus Gifu wild type, nup85-1, nup133-1, and nena-1 plants were fixed with $2.5 \%$ glutaraldehyde in fixation buffer ( $75 \mathrm{mM}$ sodium cacodylate; $2 \mathrm{mM} \mathrm{MgCl}$; $\mathrm{pH}$ 7) overnight, then washed four times in fixation buffer and postfixed for $4 \mathrm{~h}$ with $1 \%$ osmium tetroxide in fixation buffer. After two additional washing steps with fixation buffer and distilled water, the root tips were dehydrated in an acetone dilution series $(10,20,40,60,80,100 \%, 20 \mathrm{~min}$ each). For staining the $20 \%$ acetone was supplemented with $1 \%$ uranyl acetate. The samples were embedded in Spurr's low viscosity resin by a gradual infiltration series (resin/acetone: $1 / 1,2 / 1,3 / 1,4 / 1,5 / 1,4 \mathrm{~h}$ each, then $100 \%$ resin overnight) and polymerized at $63^{\circ} \mathrm{C}$. Ultrathin sections of $50-70 \mathrm{~nm}$ were cut with a diamond knife and mounted on uncoated copper grids. The sections were poststained with aqueous lead citrate $(100 \mathrm{mM}, \mathrm{pH} 13)$. Micrographes were taken with an EM 912 electron microscope (Zeiss) equipped with an integrated OMEGA energy filter operated in zero-loss mode. For each line approximately 10 individual root tips were analyzed. NPCs were quantified using Adobe Illustrator by measuring the distances between the centers of two adjacent nuclear pores.

Statistical tests were performed using R 2.15.1 software ( $R$ Core Team, 2013).

\section{RESULTS \\ LOCALIZATION OF L. japonicus NUP133 WAS UNCHANGED IN THE nup85 MUTANT}

Nuclear envelope localization of L. japonicus NUP133 was corroborated by whole-mount immunolocalization with a custom $\alpha$-NUP133 antibody. Antibody specificity was confirmed by the absence of the nuclear signal in the nup133-1 mutant (Table 1; Supplementary Figure 1). Using super-resolution 3D structured illumination microcopy (3D-SIM), NUP133 revealed a dotted localization pattern in the circumference of the nuclear rim, in accordance with its predicted function as a nucleoporin (Figure 1). Individual dots likely corresponded to single NPCs 

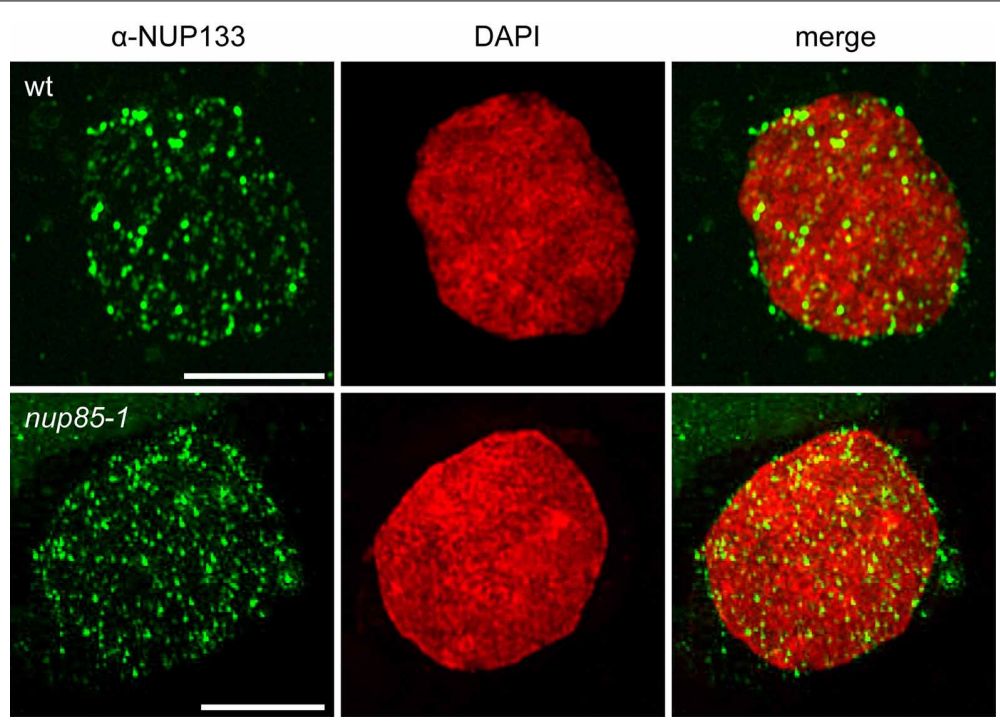

FIGURE 1 | Indirect immunolocalization of $L$. japonicus NUP133. 3D structured illumination microscopy (Z-stack) detected a punctated NUP133 signal around the nucleus, corresponding to individual NPCs or clusters of nuclear pores. No change to NUP133 localization was observed in the nup85-1 mutant. The $\alpha$-NUP133 primary antibody was visualized with an Alexa Fluor $₫ 488$ labeled secondary antibody (green). DNA was stained with DAPI (red). wt, wild type. Scale bars $=5 \mu \mathrm{m}$.
Table 1 | Analyzed L. japonicus nucleoporin mutant lines.

\begin{tabular}{|c|c|c|c|}
\hline Line & Genomic mutation & Effect & References \\
\hline nup85-1 & $\mathrm{G}_{4992}$ to $\mathrm{A}$ & $W_{306}$ to STOP & $\begin{array}{l}\text { Kistner et al., 2005; } \\
\text { Saito et al., } 2007\end{array}$ \\
\hline nup 133-1 & $\mathrm{A}_{1196}-\mathrm{T}_{1205}$ deletion & Frame shift & $\begin{array}{l}\text { Kanamori et al., } \\
2006\end{array}$ \\
\hline nena-1 & $\mathrm{C}_{400} \mathrm{~T}^{\mathrm{T}}$ & $\mathrm{Q}_{97}$ to STOP & Groth et al., 2010 \\
\hline
\end{tabular}

or NPC clusters. The observed localization was unchanged in nup85 nuclei, suggesting that the overall NPC distribution was not affected in the mutant.

\section{nup85, nup133, AND nena MUTANTS DID NOT EXHIBIT CLUSTERING OF NPCS OR ABERRATIONS IN THE NUCLEAR ENVELOPE}

We compared ultrathin sections of $L$. japonicus root tips of wild type plants with those of mutant lines nup85-1, nup1331 and nena-1 (Table 1) by transmission electron microscopy (TEM). No obvious morphological changes to the nuclear membranes or nuclear morphology could be detected beyond the intra-genotype variation (Figure 2A). NPC distribution was quantified in detail by measuring the distances between adjacent nuclear pores. The main criteria for identification of NPCs were a visible gap in the nuclear membranes, as well as a darker staining of the NPC area (Figure 2A). The overall distance distribution was comparable in mutant and wild type nuclei (Figure 2B). Average distances between pores were $533 \mathrm{~nm}$ for Gifu wild type, $599 \mathrm{~nm}$ for $n u p 85-1,440 \mathrm{~nm}$ for nup133-1 and $508 \mathrm{~nm}$ for nena-1. A multiple comparisons of means (all vs. Gifu wild type) using Dunnett contrasts identified nup133-1 as having a smaller mean than the wild type, indicating a slightly increased density of NPCs. In contrast, nup85-1 and nena-1 did not differ from the wild type (Figure 1C).

\section{LOSS OF $L$. japonicus NENA AND NUP85 REDUCED THE PROTEIN LEVELS OF OTHER PUTATIVE NUP107-160 SUBCOMPLEX MEMBERS}

Total protein extracts of Gifu wt, nup85-1, nup133-1 or nena-1 roots were probed with specific $\alpha$-NUP85 or $\alpha$-NUP133 antibodies and simultaneously with antibodies against histone $\mathrm{H} 3$. Specific NUP85 and NUP133 signals were not detected in the nup85-1 and nup133-1 extracts respectively, indicating that full length protein was absent from the mutants. However, the presence of residual truncated NUP85 and NUP133 protein could not be ruled out. The NUP85 antibody targets the carboxyterminus of the protein beyond the nup 85-1 nonsense mutation site and would therefore not bind to a truncated version. The NUP133 antibody was raised against a peptide of 21 amino acids, of which 6 amino acids are affected by the frame shift mutation in nup133-1 and might therefore also no longer detect the predicted truncated NUP133 version of 303 amino acids. In case of nena-1 it was previously indicated that the allele is a complete null (Groth et al., 2010). For NUP quantification, three independent root extracts were assayed for wild type and each mutant line, and relative protein levels of NUP85 and NUP133 were determined via normalization against histone H3 (Figure 3). No significant change relative to wild-type was observed for either NUP133 in the nena-1 background, or for NUP85 in the nup133-1 background. However, protein abundance of NUP85 in the nena-1 mutant and NUP133 in the nup $85-1$ mutant was significantly reduced to about $40 \%$ and $50 \%$ of wild type levels, respectively (Figure 3). This indicated that the loss of one nuclear pore component in L.japonicus interfered with the protein levels of other NUP107-160 sub-complex members. 


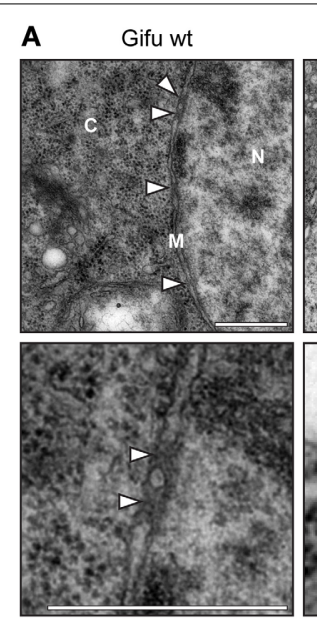

B
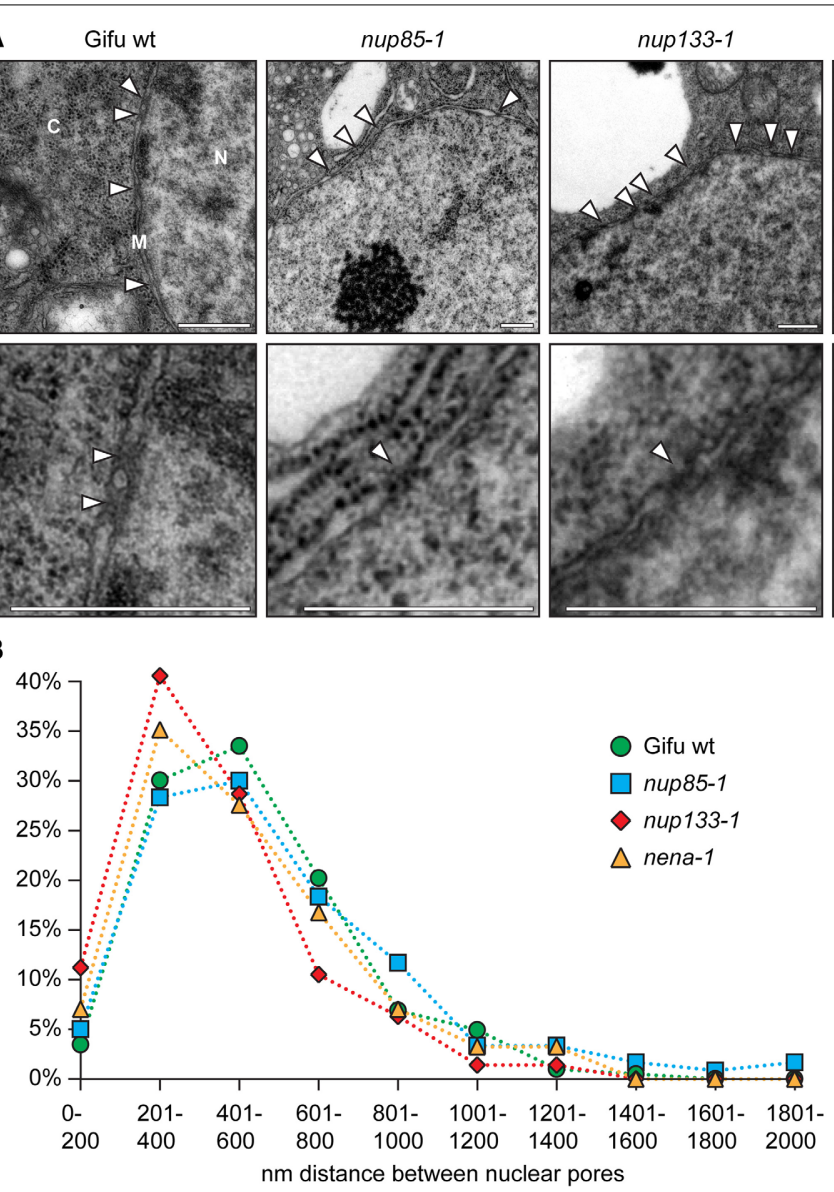

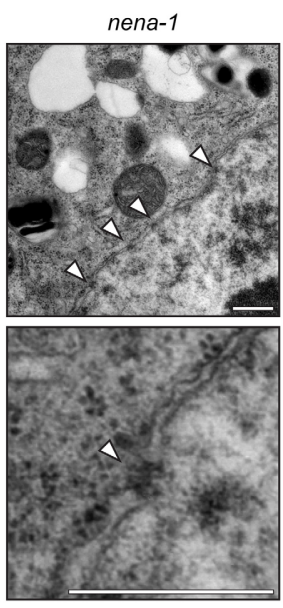

C

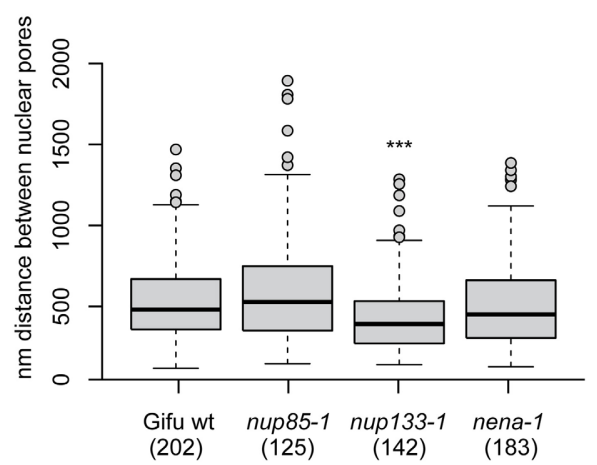

FIGURE 2 | NPC distance distribution. (A) TEM micrographs of ultrathin sections of $L$. japonicus root tips $(N$, nucleus; $C$, cytosol; $\mathrm{M}$, nuclear membranes). White arrow heads indicate the position of nuclear pores. The lower images show a magnified view of individual pores. Scale bar $=500 \mathrm{~nm}$. (B) Distance distribution between adjacent NPCs. (C) Box plot showing NPC distances.
"***" indicates significant difference from the wild type. (ANOVA revealed significant differences between average distances with $F_{(3,648)}=7.63, \quad p<0.001$. Dunnett test all vs. wild type on log transformed data identified nup133-1 as having a smaller mean than wild type; $p<0.001)$. The number of measured distances for each line is indicated in brackets below the $x$-axis.

\section{nup85/nup133 DOUBLE MUTANTS WERE SEVERELY AFFECTED IN DEVELOPMENT IN A TEMPERATURE-DEPENDENT MANNER}

To study effects associated with the complete loss of more than one nucleoporin, nup85-1 and nup133-1 mutants were crossed to generate double mutant lines. Individual F1 progeny heterozygous for both loci were identified by PCR based genotyping (not shown) and selfed. As both NUP85 and NUP133 loci are located on top of $L$. japonicus chromosome 1 in a genetic distance of $10 \mathrm{cM}$, only about $1 \%$ of the F2 offspring were expected to be homozygous nup85/nup 133 mutants (about 10\% of the gametes should carry both nup85-1 and nup133-1 mutations). Since no double mutants could be obtained in 96 analyzed F2 plants, F2 individuals that were homozygous for one mutation and heterozygous for the other were selected and again selfed. In the F3 offspring of such individuals, nup85/nup133 double mutants could be identified that showed severe and temperaturedependent developmental defects. At $24^{\circ} \mathrm{C}$, double mutants were found only among imbibed seeds that did not germinate. In contrast, homozygous nup85/nup133 plants incubated at $18^{\circ} \mathrm{C}$ were able to develop roots and cotyledons. However, they were severely impaired in growth compared to wild type and nup85 or nup133 single mutants (Figure 4). 25\% of the selected F2 progeny were expected to be homozygous for both nup85 and nup133, yet of the analyzed individuals only $7.7 \%$ ( 7 of 91 ) were double mutants and the observed allele distribution was significantly different from the expected values ( $p<0.01 ; X^{2}$ test). Previously, a reduction in seed yield was also reported in single nup 85 and nup 133 mutants as well as shorter pollen tubes in nup85 mutants (Kanamori et al., 2006; Saito et al., 2007). This suggests that nup85 and nup133 mutations affect gametophyte and seed development, resulting in fewer seeds carrying both mutations.

\section{DISCUSSION}

Here we studied the effects of mutations in genes coding for the L. japonicus NUP107-160 subcomplex members NUP85, NUP133, and NENA (SEH1). Unlike in S. cerevisiae nup85 and nup133, (Doye et al., 1994; Li et al., 1995; Siniossoglou et al., 1996), L. japonicus nup85, nup133 and nena/seh1 mutants do 


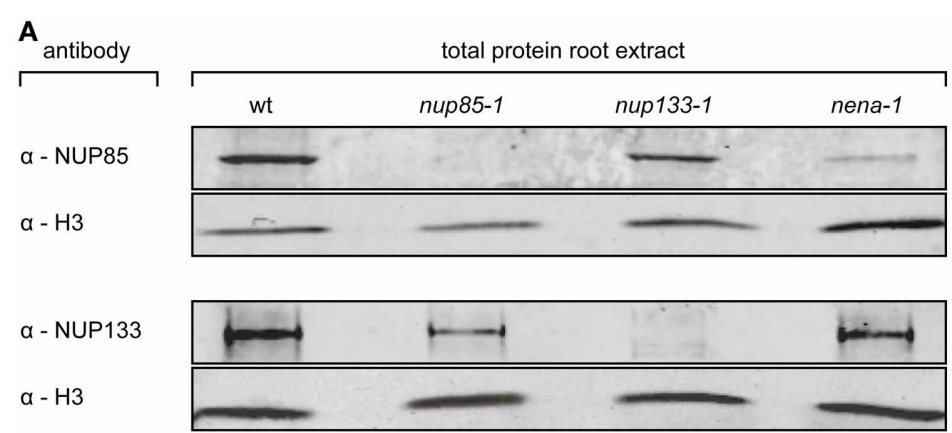

B

NUP85 protein levels

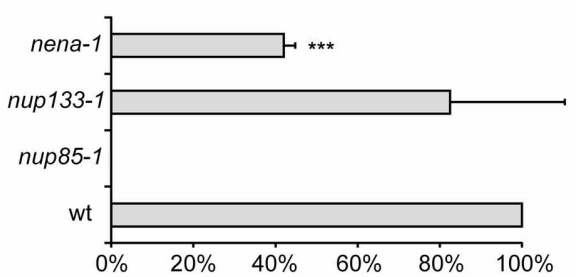

FIGURE 3 | Quantification of NUP85 and NUP133 protein levels. (A) Total protein extract from $L$. japonicus roots was separated by SDS-PAGE, blotted on membrane and immunolabelled with $\alpha$-NUP85, $\alpha$-NUP133, and $\alpha$-histone H3 antibodies. (B) For quantification, signal intensities of NUP85 and NUP133

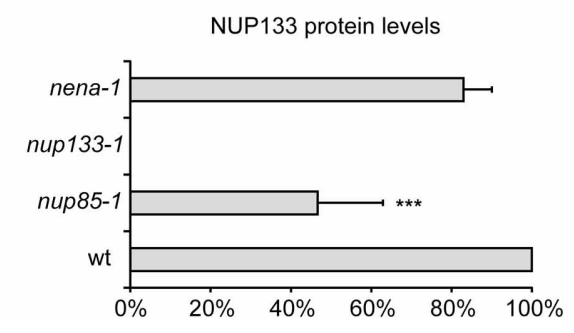

were normalized by the histone $\mathrm{H} 3$ signal and shown as percentages of wild type levels (wt). Mean protein levels were averaged from three independent experiments. Error bars show SD, "***" indicate significant differences from the wild type ( $P \leq 0.05$, $t$-test).

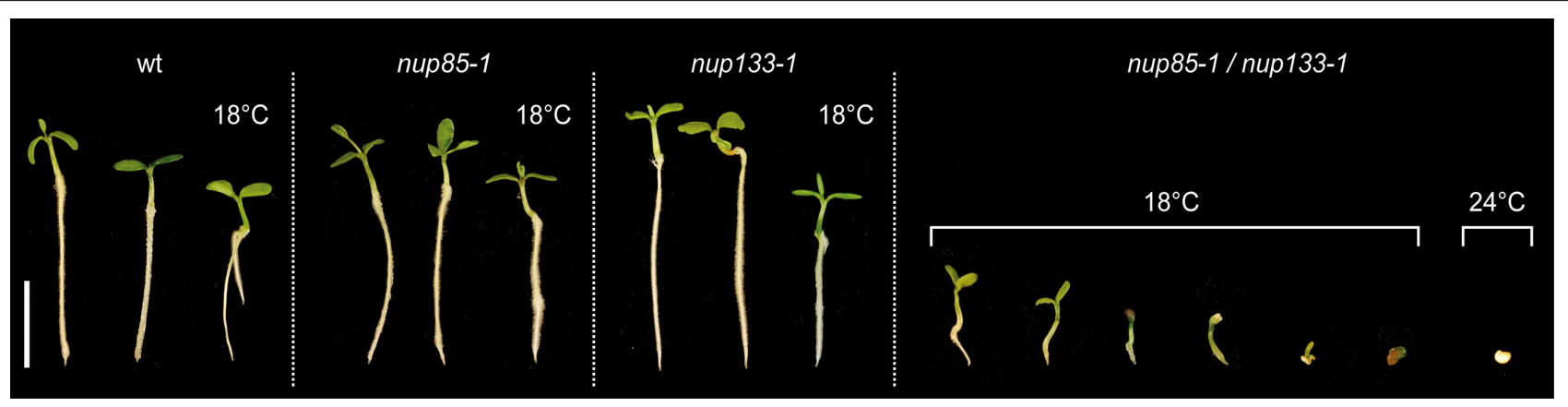

FIGURE 4 | Analysis of nup85/nup 133 double mutants. The loss of both NUP85 and NUP133 caused severe growth and developmental defects in the double mutants. At low temperature $\left(18^{\circ} \mathrm{C}\right)$ cotyledons and short roots could still develop, while at higher temperature $\left(24^{\circ} \mathrm{C}\right)$, plant development was arrested at the early germination stages. Plants and undeveloped seeds were cultivated for 16 days. Scale bar $=1 \mathrm{~cm}$. not exhibit severe alterations in NPC distribution or nuclear envelope structure, except for a decrease in the average distance of neighboring NPCs in nup133. The three L. japonicus nup mutants and $A$. thaliana nup133 and seh1 are largely indistinguishable from wild type plants (Kanamori et al., 2006; Saito et al., 2007; Groth et al., 2010; Wiermer et al., 2012), while $S$. cerevisiae nup85, nup133 and seh1 mutants show severe fitness defects (Pemberton et al., 1995; Siniossoglou et al., 1996). This lack of more prominent phenotypes at the whole plant and at the nuclear level suggests that with regard to general functions the plant NPC can tolerate the loss of single structural NUPs better than the $S$. cerevisiae NPC. Phenotypes in animal nup mutants that are not correlated with changes in nuclear pore number are often attributed to specific functions of individual
NUPs (Raices and D’Angelo, 2012). However, this is difficult to confirm, as mutations in single NUPs could affect other complex components.

Our data show that loss of L. japonicus NUP85 or NENA/SEH1 causes a reduction in the protein abundance of other putative NUP107-160 constituents. Similar effects have been previously observed in HeLa cells, where partial RNAi mediated knock-down of individual NUP107-160 NUPs (including NUP85, NUP133 and SEH1) lead to co-depletion of a number of other distinct NUPs from the nuclear envelope and in some cases to their proteolytic degradation (Boehmer et al., 2003; Harel et al., 2003; Walther et al., 2003; Loïodice et al., 2004; Rasala et al., 2006). The reduction of NUP85 levels in the L. japonicus nena mutant could be a result of the direct association of SEH1 and NUP85 
(Groth et al., 2010). In vitro experiments previously demonstrated that yeast SEC13 can form a complex with NUP85 in the absence of SEH1, suggesting a certain level of redundancy in the architecture of the NUP84 subcomplex (Debler et al., 2008). A reduced amount of soluble NUP85-SEC13 indicated that the complex was less stable than the NUP85-SEH1 interaction (Debler et al., 2008). This destabilization could explain the decrease of NUP85 levels in the Lotus nena-1 mutant, assuming that NENA/SEH1 was replaced by SEC13. As the NUP85-SEH1 interaction is structurally very similar to the NUP145C-SEC13 pair (Hsia et al., 2007), it is also conceivable that the loss of NENA causes a partial displacement of NUP85 by NUP145-SEC13, which in turn could lead to the increased degradation of NUP85. The fact that NUP85 levels were not reduced in the nup133-1 mutant, but NUP133 was reduced in nup85-1 could potentially be due to residual truncated NUP133 protein that was not detected by the antibodies. In yeast, NUP133 and NUP85 are positioned at opposite ends of the Y shaped NUP84 complex and do not directly interact (Lutzmann et al., 2002; Brohawn et al., 2008; Fernandez-Martinez et al., 2012). The reduction of NUP133 in the nup 85 mutant therefore suggests a more widespread alteration of the NUP107-160 complex. It is likely that the shared phenotypes in symbiotic signaling in nup85, nup133 and nena are due the changes in the subcomplex and are not a function of the individual nucleoporins. In yeast mutants, NPC clustering and fitness phenotypes were shown not to correlate in severity, but instead could be mapped to different NUP84 subcomplex regions (Fernandez-Martinez et al., 2012). In the case of L. japonicus, it would also be interesting to analyze other NUP107-160 subcomplex mutants, with regard to both general phenotypes as well as symbiotic defects, in order to pinpoint potential distinct functional areas of the subcomplex.

There is accumulating evidence that NPCs are more heterogeneous in their composition than previously assumed (Raices and D’Angelo, 2012). In animals, several NUPs, including NUP133 are differentially expressed in different cell types and tissues (Zhang et al., 1999; Guan et al., 2000; Olsson et al., 2004; Lupu et al., 2008; Cho et al., 2009) and mutations in multiple NUPs were correlated with tissue-specific diseases (Huebner et al., 2004; Basel-Vanagaite et al., 2006; Zhang et al., 2008). Additionally over one third of all nucleoporins are present in varying stoichiometries in different cell types, including the NUP107-160 subcomplex members SEH1 and ELYS, suggesting that distinct versions of the subcomplex exist (Ori et al., 2013). This flexibility in nuclear pore composition potentially allows for stable NPC configurations even in the absence of individual NUPs, however certain functions can potentially no longer be fully performed.

The fact that nup85/nup133 double mutants have more severe general phenotypes than either of the single mutants, highlights the functional overlap of the proteins and suggests that loss of more than one NUP107-160 complex component leads to a break-down of basal NPC functions. Stronger phenotypes have also been reported in double mutants of $A$. thaliana nup96 and nup160 (Parry et al., 2006) as well as nup133 and nup160 (Wiermer et al., 2012). Additionally in S. cerevisiae most combinations of double mutants affecting NUP107-160 complex components are synthetically lethal (Siniossoglou et al., 1996). In vitro experiments with reconstituted Xenopus nuclei have previously shown that depletion of NUP85 and NUP133 completely prevented the assembly of the NUP107-160 subcomplex (Harel et al., 2003). It is therefore likely that in the L. japonicus nup85/nup133 double mutants the subcomplex, and in extension the NPC as a whole, are strongly affected. However, since cell division occurs, cellular functions seem to be maintained to some extent, making it unlikely that the NPC cannot assemble at all.

The symbiotic phenotypes in L. japonicus nup85, nup133 and nena mutants (Kanamori et al., 2006; Saito et al., 2007; Groth et al., 2010) and the growth defects in several yeast nup mutants, including nup133 and nup85 (Doye et al., 1994; Li et al., 1995; Siniossoglou et al., 1996; Fernandez-Martinez et al., 2012) are less pronounced at lower temperatures. We demonstrated that a shift from 24 to $18^{\circ} \mathrm{C}$ also reduces the severity of the developmental defects in the Lotus nup85/nup133 mutants. This suggests that depending on low temperature, the plant NPC can even tolerate the loss of more than one structural nuclear pore component to a certain degree. The temperature dependence both in the single and double mutants could indicate that functional redundancy is provided by an imperfect fit of alternate NUP-NUP interactions, which are destabilized at higher temperatures.

Our data supports the view of a flexible and dynamic composition of the NPC. A certain level of redundancy in the NPC architecture can potentially compensate for the loss of even structural NUPs such as NUP85, NUP133 and NENA/SEH1 with regard to many general NPC functions in particular at lower temperatures. At the same time certain pathways, such as symbiotic signaling, that may be susceptible to quantitative changes in NPC transport capabilities, can be affected by alterations in the NPC composition or by the lack of particular NPC confirmations.

\section{ACKNOWLEDGMENTS}

For help with the statistical analysis we thank Dr. Axel Strauß (LMU Munich). We are grateful to Dr. Lothar Schermelleh, now at the University of Oxford, for assistance with super-resolution microcopy and usage of the DeltaVision OMX microscope. We also thank Sylvia Dobler (LMU Munich) for providing help with sample preparation for electron microscopy. We thank the German Research Foundation for continuous support.

\section{SUPPLEMENTARY MATERIAL}

The Supplementary Material for this article can be found online at: http://www.frontiersin.org/journal/10.3389/fpls.2013. 00552/abstract

\section{REFERENCES}

Aitchison, J. D., Blobel, G., and Rout, M. P. (1995). Nup120p: a yeast nucleoporin required for NPC distribution and mRNA transport. J. Cell Biol. 131, 1659-1675. doi: 10.1083/jcb.131.6.1659

Aitchison, J. D., and Rout, M. P. (2012). The yeast nuclear pore complex and transport through it. Genetics 190, 855-883. doi: 10.1534/genetics.111.127803

Alber, F., Dokudovskaya, S., Veenhoff, L. M., Zhang, W., Kipper, J., Devos, D., et al. (2007). The molecular architecture of the nuclear pore complex. Nature 450, 695-701. doi: 10.1038/nature06405

Basel-Vanagaite, L., Muncher, L., Straussberg, R., Pasmanik-Chor, M., Yahav, M., Rainshtein, L., et al. (2006). Mutated nup62 causes autosomal recessive infantile bilateral striatal necrosis. Ann. Neurol. 60, 214-222. doi: 10.1002/ ana.20902 
Belgareh, N., Rabut, G., Baï, S. W., Van Overbeek, M., Beaudouin, J., Daigle, N., et al. (2001). An evolutionarily conserved NPC subcomplex, which redistributes in part to kinetochores in mammalian cells. J. Cell Biol. 154, 1147-1160. doi: 10.1083/jcb.200101081

Binder, A., and Parniske, M. (2013). "The nuclear pore complex in symbiosis and pathogen defence," in Annual Plant Reviews, eds D. E. Evans, K. Graumann, and J. A. Bryant (Oxford: John Wiley \& Sons Ltd.), 229-254.

Boehmer, T., Enninga, J., Dales, S., Blobel, G., and Zhong, H. (2003). Depletion of a single nucleoporin, Nup107, prevents the assembly of a subset of nucleoporins into the nuclear pore complex. Proc. Natl. Acad. Sci. U.S.A. 100, 981-985. doi: 10.1073/pnas. 252749899

Brohawn, S. G., Leksa, N. C., Spear, E. D., Rajashankar, K. R., and Schwartz, T. U. (2008). Structural evidence for common ancestry of the nuclear pore complex and vesicle coats. Science 322, 1369-1373. doi: 10.1126/science.1165886

Bukata, L., Parker, S. L., and D'angelo, M. A. (2013). Nuclear pore complexes in the maintenance of genome integrity. Curr. Opin. Cell Biol. 25, 378-386. doi: 10.1016/j.ceb.2013.03.002

Capelson, M., Doucet, C., and Hetzer, M. W. (2010). Nuclear pore complexes: guardians of the nuclear genome. Cold Spring Harb. Symp. Quant. Biol. 75, 585-597. doi: 10.1101/sqb.2010.75.059

Capelson, M., and Hetzer, M. W. (2009). The role of nuclear pores in gene regulation, development and disease. EMBO Rep. 10, 934-934. doi: 10.1038/embor.2009.176

Cheng, Y. T., Germain, H., Wiermer, M., Bi, D., Xu, F., García, A. V., et al. (2009). Nuclear pore complex component MOS7/Nup88 is required for innate immunity and cuclear accumulation of defense regulators in Arabidopsis. Plant Cell 21, 2503-2516. doi: 10.1105/tpc.108.064519

Cho, A. R., Yang, K.-J., Bae, Y., Bahk, Y. Y., Kim, E., Lee, H., et al. (2009). Tissuespecific expression and subcellular localization of ALADIN, the absence of which causes human triple A syndrome. Exp. Mol. Med. 41, 381-386. doi: 10.3858/emm.2009.41.6.043

Debler, E. W., Ma, Y., Seo, H.-S., Hsia, K.-C., Noriega, T. R., Blobel, G., et al. (2008). A fence-like coat for the nuclear pore membrane. Mol. Cell 32, 815-826. doi: 10.1016/j.molcel.2008.12.001

Dong, C.-H., Hu, X., Tang, W., Zheng, X., Kim, Y. S., Lee, B.-H., et al. (2006). A putative Arabidopsis nucleoporin, AtNUP160, Is critical for RNA export and required for plant tolerance to cold stress. Mol. Cell. Biol. 26, 9533-9543. doi: 10.1128/MCB.01063-06

Doucet, C. M., Talamas, J. A., and Hetzer, M. W. (2010). Cell cycle-dependent differences in nuclear pore complex assembly in metazoa. Cell 141, 1030-1041. doi: 10.1016/j.cell.2010.04.036

Doye, V., Wepf, R., and Hurt, E. C. (1994). A novel nuclear pore protein Nup133p with distinct roles in poly(A)+ RNA transport and nuclear pore distribution. EMBO J. 13, 6062-6075.

Faria, A. M. C., Levay, A., Wang, Y., Kamphorst, A. O., Rosa, M. L. P., Nussenzveig, D. R., et al. (2006). The nucleoporin Nup96 Is required for proper expression of interferon-regulated proteins and functions. Immunity 24, 295-304. doi: 10.1016/j.immuni.2006.01.014

Fernandez-Martinez, J., Phillips, J., Sekedat, M. D., Diaz-Avalos, R., VelazquezMuriel, J., Franke, J. D., et al. (2012). Structure-function mapping of a heptameric module in the nuclear pore complex. J. Cell Biol. 196, 419-434. doi: 10.1083/jcb. 201109008

Fiserova, J., Kiseleva, E., and Goldberg, M. W. (2009). Nuclear envelope and nuclear pore complex structure and organization in tobacco BY-2 cells. Plant J. 59, 243-255. doi: 10.1111/j.1365-313X.2009.03865.x

Goldberg, M. W., and Allen, T. D. (1996). The nuclear pore complex and lamina: three-dimensional structures and interactions determined by field emission in-lens scanning electron microscopy. J. Mol. Biol. 257, 848-865. doi: 10.1006/jmbi.1996.0206

Grossman, E., Medalia, O., and Zwerger, M. (2012). Functional architecture of the nuclear pore complex. Annu. Rev. Biophys. 41, 557-584. doi: 10.1146/annurevbiophys-050511-102328

Groth, M., Takeda, N., Perry, J., Uchida, H., Dräxl, S., Brachmann, A., et al. (2010). NENA, a Lotus japonicus homolog of Sec13, is required for rhizodermal infection by arbuscular mycorrhiza fungi and rhizobia but dispensable for cortical endosymbiotic development. Plant Cell 22, 2509-2526. doi: 10.1105/tpc.109.069807

Guan, T., Kehlenbach, R. H., Schirmer, E. C., Kehlenbach, A., Fan, F., Clurman, B. E., et al. (2000). Nup50, a nucleoplasmically oriented nucleoporin with a role in nuclear protein export. Mol. Cell. Biol. 20, 5619-5630. doi: 10.1128/MCB.20.15.5619-5630.2000

Harel, A., Orjalo, A. V., Vincent, T., Lachish-Zalait, A., Vasu, S., Shah, S., et al. (2003). Removal of a single pore subcomplex results in vertebrate nuclei devoid of nuclear pores. Mol. Cell 11, 853-864. doi: 10.1016/S1097-2765(03) 00116-3

Heath, C. V., Copeland, C. S., Amberg, D. C., Del Priore, V., Snyder, M., and Cole, C. N. (1995). Nuclear pore complex clustering and nuclear accumulation of poly(A)+ RNA associated with mutation of the Saccharomyces cerevisiae RAT2/NUP120 gene. J. Cell Biol. 131, 1677-1697. doi: 10.1083/jcb.131. 6.1677

Hoelz, A., Debler, E. W., and Blobel, G. (2011). The structure of the nuclear pore complex. Annu. Rev. Biochem. 80, 613-643. doi: 10.1146/annurev-biochem060109-151030

Hsia, K.-C., Stavropoulos, P., Blobel, G., and Hoelz, A. (2007). Architecture of a coat for the nuclear pore membrane. Cell 131, 1313-1326. doi: 10.1016/j.cell.2007.11.038

Huebner, A., Kaindl, A. M., Knobeloch, K. P., Petzold, H., Mann, P., and Koehler, K. (2004). The triple a syndrome Is due to mutations in ALADIN, a novel member of the nuclear pore complex. Endo. Res. 30, 891-899. doi: 10.1081/ERC200044138

Jiang, Q., and Gresshoff, P. M. (1997). Classical and molecular genetics of the model legume Lotus japonicus. Mol. Plant Microbe Interact. 10, 59-68. doi: 10.1094/MPMI.1997.10.1.59

Kanamori, N., Madsen, L. H., Radutoiu, S., Frantescu, M., Quistgaard, E. M., Miwa, H., et al. (2006). A nucleoporin is required for induction of $\mathrm{Ca}^{2+}$ spiking in legume nodule development and essential for rhizobial and fungal symbiosis. Proc. Natl. Acad. Sci. U.S.A. 103, 359-364. doi: 10.1073/pnas.0508883103

Kiseleva, E., Allen, T. D., Rutherford, S., Bucci, M., Wente, S. R., and Goldberg, M. W. (2004). Yeast nuclear pore complexes have a cytoplasmic ring and internal filaments. J. Struct. Biol. 145, 272-288. doi: 10.1016/j.jsb.2003.11.010

Kistner, C., Winzer, T., Pitzschke, A., Mulder, L., Sato, S., Kaneko, T., et al. (2005). Seven Lotus japonicus genes required for transcriptional reprogramming of the root during fungal and bacterial symbiosis. Plant Cell 17, 2217-2229. doi: 10.1105/tpc.105.032714

Li, O., Heath, C. V., Amberg, D. C., Dockendorff, T. C., Copeland, C. S., Snyder, M., et al. (1995). Mutation or deletion of the Saccharomyces cerevisiae RAT3/NUP133 gene causes temperature-dependent nuclear accumulation of poly(A)+ RNA and constitutive clustering of nuclear pore complexes. Mol. Biol. Cell 6, 401-417. doi: 10.1091/mbc.6.4.401

Loïodice, I., Alves, A., Rabut, G., Van Overbeek, M., Ellenberg, J., Sibarita, J.-B., et al. (2004). The entire Nup107-160 Complex, including three new members, is targeted as one entity to kinetochores in mitosis. Mol. Biol. Cell 15, 3333-3344. doi: 10.1091/mbc.E03-12-0878

Lupu, F., Alves, A., Anderson, K., Doye, V., and Lacy, E. (2008). Nuclear pore composition regulates neural stem/progenitor cell differentiation in the mouse embryo. Dev. Cell 14, 831-842. doi: 10.1016/j.devcel.2008.03.011

Lutzmann, M., Kunze, R., Buerer, A., Aebi, U., and Hurt, E. (2002). Modular selfassembly of a Y-shaped multiprotein complex from seven nucleoporins. EMBO J. 21, 387-397. doi: 10.1093/emboj/21.3.387

Olsson, M., Schéele, S., and Ekblom, P. (2004). Limited expression of nuclear pore membrane glycoprotein 210 in cell lines and tissues suggests celltype specific nuclear pores in metazoans. Exp. Cell Res. 292, 359-370. doi: 10.1016/j.yexcr.2003.09.014

Ori, A., Banterle, N., Iskar, M., Andres-Pons, A., Escher, C., Khanh Bui, H., et al. (2013). Cell type-specific nuclear pores: a case in point for contextdependent stoichiometry of molecular machines. Mol. Syst. Biol. 9:648. doi: 10.1038/msb.2013.4

Orjalo, A. V., Arnaoutov, A., Shen, Z., Boyarchuk, Y., Zeitlin, S. G., Fontoura, B., et al. (2006). The Nup107-160 nucleoporin complex is required for correct bipolar spindle assembly. Mol. Biol. Cell 17, 3806-3818. doi: 10.1091/mbc.E0511-1061

Parry, G., Ward, S., Cernac, A., Dharmasiri, S., and Estelle, M. (2006). The Arabidopsis SUPPRESSOR OF AUXIN RESISTANCE proteins are nucleoporins with an important role in hormone signaling and development. Plant Cell 18, 1590-1603. doi: 10.1105/tpc.106.041566

Pemberton, L. F., Rout, M. P., and Blobel, G. (1995). Disruption of the nucleoporin gene NUP133 results in clustering of nuclear pore complexes. Proc. Natl. Acad. Sci. U.S.A. 92, 1187-1191. doi: 10.1073/pnas.92.4.1187 
R. Core Team (2013). R: A Language and Environment for Statistical Computing [Online]. $R$ Foundation for Statistical Computing. Available online at: http://www.R-project.org

Raices, M., and D’Angelo, M. A. (2012). Nuclear pore complex composition: a new regulator of tissue-specific and developmental functions. Nat. Rev. Mol. Cell Biol. 13, 687-699. doi: 10.1038/nrm3461

Rasala, B. A., Orjalo, A. V., Shen, Z., Briggs, S., and Forbes, D. J. (2006). ELYS is a dual nucleoporin/kinetochore protein required for nuclear pore assembly and proper cell division. Proc. Natl. Acad. Sci. 103, 17801-17806. doi: 10.1073/pnas.0608484103

Robles, L. M., Deslauriers, S. D., Alvarez, A. A., and Larsen, P. B. (2012). A loss-of-function mutation in the nucleoporin AtNUP160 indicates that normal auxin signalling is required for a proper ethylene response in Arabidopsis. J. Exp. Bot. 63, 2231-2241. doi: 10.1093/ jxb/err424

Saito, K., Yoshikawa, M., Yano, K., Miwa, H., Uchida, H., Asamizu, E., et al. (2007). NUCLEOPORIN85 is required for calcium spiking, fungal and bacterial symbioses, and seed production in Lotus japonicus. Plant Cell 19, 610-624. doi: 10.1105/tpc.106.046938

Sauer, M., Paciorek, T., Benkova, E., and Friml, J. (2006). Immunocytochemical techniques for whole-mount in situ protein localization in plants. Nat. Protoc. 1, 98-103. doi: 10.1038/nprot.2006.15

Siniossoglou, S., Wimmer, C., Rieger, M., Doye, V., Tekotte, H., Weise, C., et al. (1996). A novel complex of nucleoporins, which Includes sec13p and a sec13p homolog, is essential for normal nuclear pores. Cell 84, 265-275. doi: 10.1016/S0092-8674(00)80981-2

Strambio-De-Castillia, C., Niepel, M., and Rout, M. P. (2010). The nuclear pore complex: bridging nuclear transport and gene regulation. Nat. Rev. Mol. Cell Biol. 11, 490-501. doi: 10.1038/nrm2928

Tamura, K., Fukao, Y., Iwamoto, M., Haraguchi, T., and Hara-Nishimura, I. (2010). Identification and characterization of nuclear pore complex components in Arabidopsis thaliana. Plant Cell 22, 4084-4097. doi: 10.1105/tpc.110. 079947

Van De Vosse, D. W., Wan, Y., Lapetina, D. L., Chen, W.-M., Chiang, J.-H., Aitchison, J D., et al. (2013). A role for the nucleoporin Nup170p in chromatin structure and gene silencing. Cell 152, 969-983. doi: 10.1016/j.cell.2013. 01.049

Van De Vosse, D. W., Wan, Y., Wozniak, R. W., and Aitchison, J. D. (2011). Role of the nuclear envelope in genome organization and gene expression. Wiley Interdiscip. Rev. Syst. Biol. Med. 3, 147-166. doi: 10.1002/ wsbm.101
Walther, T. C., Alves, A., Pickersgill, H., Lol Odice, I., Hetzer, M., Galy, V., et al. (2003). The conserved Nup107-160 complex is critical for nuclear pore complex assembly. Cell 113, 195-206. doi: 10.1016/S0092-8674(03)00235-6

Wiermer, M., Cheng, Y. T., Imkampe, J., Li, M., Wang, D., Lipka, V., et al. (2012). Putative members of the Arabidopsis Nup107-160 nuclear pore sub-complex contribute to pathogen defense. Plant J. 70, 796-808. doi: 10.1111/j.1365313X.2012.04928.x

Wozniak, R., Burke, B., and Doye, V. (2010). Nuclear transport and the mitotic apparatus: an evolving relationship. Cell. Mol. Life Sci. 67, 2215-2230. doi: 10.1007/s00018-010-0325-7

Zhang, X., Chen, S., Yoo, S., Chakrabarti, S., Zhang, T., Ke, T., et al. (2008). Mutation in nuclear pore component NUP155 leads to atrial fibrillation and early sudden cardiac death. Cell 135, 1017-1027. doi: 10.1016/j.cell.2008.10.022

Zhang, X., Yang, H., Corydon, M. J., Zhang, X., Pedersen, S., Korenberg, J. R., et al. (1999). Localization of a human nucleoporin 155 gene (NUP155) to the 5 p13 region and cloning of its cDNA. Genomics 57, 144-151. doi: 10.1006/geno.1999.5741

Zhang, Y., and Li, X. (2005). A putative nucleoporin 96 is required for both basal defense and constitutive resistance responses mediated by suppressor of npr11,constitutive 1. Plant Cell 17, 1306-1316. doi: 10.1105/tpc.104.029926

Zuccolo, M., Alves, A., Galy, V., Bolhy, S., Formstecher, E., Racine, V., et al. (2007) The human Nup107-160 nuclear pore subcomplex contributes to proper kinetochore functions. EMBO J. 26, 1853-1864. doi: 10.1038/sj.emboj.7601642

Conflict of Interest Statement: The authors declare that the research was conducted in the absence of any commercial or financial relationships that could be construed as a potential conflict of interest.

Received: 04 December 2013; paper pending published: 18 December 2013; accepted: 23 December 2013; published online: 22 January 2014.

Citation: Binder A and Parniske M (2014) Analysis of the Lotus japonicus nuclear pore NUP107-160 subcomplex reveals pronounced structural plasticity and functional redundancy. Front. Plant Sci. 4:552. doi: 10.3389/fpls.2013.00552

This article was submitted to Plant Cell Biology, a section of the journal Frontiers in Plant Science.

Copyright (c) 2014 Binder and Parniske. This is an open-access article distributed under the terms of the Creative Commons Attribution License (CC BY). The use, distribution or reproduction in other forums is permitted, provided the original author(s) or licensor are credited and that the original publication in this journal is cited, in accordance with accepted academic practice. No use, distribution or reproduction is permitted which does not comply with these terms. 